\title{
Situação de Risco para Úlceras por Pressão em uma Unidade de Assistência Domiciliar
}

\author{
Risk Situation for Pressure Ulcers in Home Care Unit
}

Situación de los Riesgos para Úlceras de Presión en una Unidad de Asistencia Familiar

Edson Maruyama Diniz', Ana Beatriz Pinto da Silva Morita², Maria Angela Boccara de Paula ${ }^{3}$

\begin{abstract}
RESUMO
Estudo exploratório, descritivo e quantitativo com o objetivo de caracterizar o perfil dos pacientes atendidos pela Unidade de Assistência Domiciliar no município de São Paulo e analisar os fatores de risco relacionados ao desenvolvimento de úlceras por pressão (UP). A população foi composta por pacientes adultos totalmente dependentes com pontuação 5 na Escala de Avaliação de Incapacidades Funcionais da Cruz Vermelha Espanhola. Realizou-se coleta de dados através de instrumento composto de formulário para identificação do perfil epidemiológico e de morbidade do paciente e a aplicação da Escala de Braden. Pesquisa foi aprovada pelo comitê de ética da Unitau (n 390/12) e autorizado pela Secretaria Municipal Saúde de São Paulo. A coleta de dados foi realizada por meio de aplicação de instrumento de coleta. Dos 98 pacientes 57 eram do gênero feminino e 67 da raça branca. Sessenta participantes eram idosos e destes, 20 eram octagenários. Quase a totalidade (90) eram cuidados por familiares e todos os pacientes apresentaram algum tipo de incontinência. Houve predomínio das doenças do sistema nervoso (43) seguida pelas doenças do sistema circulatório (36). Dezesseis pacientes apresentavam UP. O escorre mínimo da escala de Braden foi de 8 pontos e o máximo de 18 pontos, sendo valor médio de 13,04 ( $\mathrm{DP}=2,3634)$. Sete pacientes apresentaram Altíssimo Risco, 36 apresentaram Risco Alto, 30 apresentaram Risco Moderado e 25 apresentaram Risco Baixo. Todos os pacientes apresentaram risco para desenvolvimento de UP. Este risco pode ser explicado devido às doenças de base dos pacientes e a presença de incontinência anal e/ou urinária.
\end{abstract}

DESCRITORES: Estomaterapia. Enfermagem. Úlcera por pressão. Perfil de saúde.

\begin{abstract}
Exploratory, descriptive and quantitative study aimed to identify the profile of patients seen by the Home Care Unit in São Paulo and analyze the risk factors related to the development of pressure ulcers (PU). The population comprised entirely dependent patients with score 5 in Assessment Scale Functional Impairments Spanish Red Cross. Data collection was carried out through an instrument composed form to identify the epidemiology and morbidity of the patient and the application of the Braden Scale. Study was approved by the ethics committee of Unitau (No. 390/12) and approved by the Municipal Health Secretary of São Paulo. Data collection was performed by application of data collection instrument. Of the 98 patients 57 were female and 67 gender Caucasians. Sixty participants were elderly and of these, 20 were octogenarians. Almost all (90) were cared for by relatives and all patients had some type of incontinence. There was a predominance of the nervous system (43) followed by diseases of the circulatory system (36) diseases. Sixteen patients had PU. The minimum flows Braden Scale was 8 points and a maximum of 18 points, with a mean value of 13.04 (SD = 2.3634). Seven patients had very high risk, 36 had High Risk, Moderate Risk, and 30 had had 25 Light risk. All patients were at risk for PU development. This risk can be explained due to underlying diseases of patients and the presence of anal and / or urinary incontinence.
\end{abstract}

DESCRIPTORS: Stomatherapy. Nursing. Pressure ulcer. Health profile.

'Prefeitura Municipal de São Paulo - São Paulo (SP), Brasil.

Endereço para correspondência: Avenida Sargento Geraldo Santana, 683, bloco 3, apto. 33 - Jardim Taquaral - CEP: 04674-225 - São Paulo (SP),

Brasil. E-mail: edson.marudiniz@gmail.com

${ }^{2}$ Faculdades Integradas Teresa D'Ávila (FATEA) - Lorena (SP), Brasil.

¿Universidade de Taubaté (UNITAU) - Taubaté (SP), Brasil.

Parte da Monografia intitulada "Situação de risco para úlcera por pressão em uma unidade de assistência domiciliar no munícipio de São Paulo" apresentada em 2014 pelo aluno Edson Maruyama Diniz ao Programa de Pós-Graduação Latu Sensu em Enfermagem em Estomaterapia da Universidade de Taubaté, como requisito parcial para a obtenção do título de especialista em Estomaterapia. 


\section{RESUMEN}

Estudio exploratorio, descriptivo y cuantitativo tuvo como objetivo identificar el perfil de los pacientes atendidos por la Unidad de Cuidados en el hogar, en São Paulo y analizar los factores de riesgo relacionados con el desarrollo de las úlceras de presión (UP). La población está constituida por pacientes totalmente dependientes con puntuación de 5 en la Escala de Evaluación de deficiencias funcionales de la Cruz Roja Española. La recolección de datos se llevó a cabo a través de una forma integrada de instrumentos para identificar la epidemiología y la morbilidad del paciente y la aplicación de la Escala de Braden. El estudio fue aprobado por el comité de ética de Unitau (n³90/12) y aprobado por el Secretario Municipal de Salud de São Paulo. La recolección de datos se realizó mediante la aplicación de instrumentos de recolección de datos. De los 98 pacientes 57 eran mujeres y 67 caucásicos de género. Sesenta participantes fueron personas de edad avanzada y de éstos, 20 eran octogenarios. Casi todos (90) fueron atendidos por sus familiares y todos los pacientes tenían algún tipo de incontinencia. Hubo un predominio del sistema nervioso (43), seguida de las enfermedades del sistema circulatorio (36) Enfermedades. Dieciséis pacientes presentaron UP. El mínimo fluye Escala de Braden fue de 8 puntos y un máximo de 18 puntos, con un valor medio de 13,04 (SD=2,3634). Siete pacientes tenían un riesgo muy alto, 36 tenían alto riesgo, riesgo moderado y 30 habían tenido 25 Riesgo Ligero. Todos los pacientes se encontraban en situación de riesgo para el desarrollo de la PU. Este riesgo se puede explicar debido a enfermedades subyacentes de los pacientes y la presencia de anal y / o incontinencia urinaria.

DESCRIPTORES: Estomaterapia. Enfermeria. Úlcera por presión. Perfil de Salud.

\section{INTRODUÇÃO}

O envelhecimento populacional é um fenômeno mundial que desafia a enfermagem e os serviços de saúde. Entre 1980 e 2000, a população com 60 anos ou mais cresceu 7,3 milhões de pessoas, totalizando mais de 14,5 milhões de pessoas em 2000. As projeções da Organização Mundial de Saúde (OMS) apontam que, até 2025, o Brasil será o sexto país do mundo em número de idosos, devendo ter cerca de 32 milhões de pessoas com mais de 60 anos $^{1}$.

O aumento da expectativa de vida, logrado graças aos avanços socioeconômicos e científicos, podem resultar em uma sobrevida marcada por incapacidades e dependência de terceiros. Houve uma alteração epidemiológica que resultou em uma transição demográfica, modificando o perfil de morbimortalidade da população; contudo, nem sempre essas mudanças foram acompanhadas da adequada manutenção da saúde desta clientela. Desta forma, houve o aumento da prevalência de doenças crônicas não transmissíveis (DCNT), cujas sequelas e complicações podem ocasionar incapacidades, dependência e necessidades de cuidado de longa permanência ${ }^{2}$.

Frente a estas alterações, a Assistência Domiciliar ressurge no panorama brasileiro, como uma alternativa para o tratamento destes pacientes. Esta é uma prática que pode reduzir custos hospitalares e humanizar as práticas de saúde, uma vez que as DCNT associadas ao envelhecimento populacional geram limitações que potencializam o desenvolvimento de incapacidades funcionais permanentes ou transitórias. Além disso, promove um novo conceito de gerenciamento dos recursos hospitalares públicos, uma vez que promove uma alternativa a intervenção hospitalar, graças a incorporação de novos métodos terapêuticos e tecnologias que possibilitaram que muitos tratamentos fossem realizados em regime domiciliar ${ }^{3}$.

Os pacientes submetidos a cuidados intensivos e que necessitam de elevados níveis de dependência apresentam, geralmente, alto risco para desenvolver úlceras por pressão (UP), devido a limitações ambientais e psicobiológicas, tais como: instabilidade hemodinâmica, restrição de movimentos por período prolongado de tempo e uso de drogas sedativas e analgésicas, as quais diminuem a percepção sensorial e prejudicam a mobilidade 4 .

A UP é um sério problema nas instituições hospitalares e asilares e no domicílio e depois de instalada ocasiona várias dificuldades e custos no seu tratamento tanto para o paciente, quanto para seus cuidadores e serviços de saúde. Pode ser definida como uma área localizada de morte tecidual que se desenvolve quando um tecido mole é comprimido entre uma proeminência óssea e uma superfície dura, por um periodo prolongado de tempo. Ocorre a interrupção do fornecimento do sangue para a área da lesão, provocada, sobretudo pela pressão exercida. Outras forças também podem atuar como a friç̧ão e o cisalhamento ou uma associação entre os três fatores 5 . 
As UP são um problema relevante de saúde pública, resultando em hospitalização, institucionalização, perda de qualidade de vida e alto custo de tratamento ${ }^{5}$. As UP influenciam drasticamente o período de hospitalização com repercussões diretas no desconforto e dor ocasionados aos pacientes acometidos por estas lesões ${ }^{6}$.

Assim, é importante para o enfermeiro compreender as respostas do indivíduo com risco para o desenvolvimento das UP visando uma melhoria da assistência profissional assim como a autonomia e a garantia do exercício profissional ${ }^{7}$. Tem-se percebido através da prática profissional que os pacientes atendidos pela assistência domiciliar têm um alto risco para o desenvolvimento das UP.

\section{OBJETIVOS}

Este estudo teve como objetivo caracterizar o perfil de pacientes totalmente dependentes atendidos em uma Unidade de Assistência Domiciliar no município de São Paulo (SP) e analisar os fatores de risco relacionados ao desenvolvimento de úlceras por pressão, bem como as condições de incapacidades funcionais e classi ficar o grau de risco para UP, por meio da aplicação da escala de Braden.

\section{MÉTODO}

Estudo exploratório, descritivo com abordagem quantitativa, realizada em uma Unidade de Assistência Domiciliar no município de São Paulo. A população do estudo foi composta pelos pacientes adultos, totalmente dependentes, atendidos pela Unidade de Assistência Domiciliar com pontuação 5 na Escala de Avaliação de Incapacidades Funcionais da Cruz Vermelha Espanhola para realização das Atividades de Vida Diária (AVD). Os pacientes que tiverem pontuações menores que Grau $V$ na referida escala foram excluídos do estudo.

A coleta de dados foi realizada pelo enfermeiro da unidade, com utilização de instrumento de coleta composto por duas partes. A primeira constava de um formulário para identi cação do perfil epidemiológico e de morbidade do paciente. A segunda parte incluiu a observação do paciente para avaliação de suas condições de incapacidades funcionais e quadro clínico, objetivando a avaliação de risco para o desenvolvimento das UP, sendo realizada por meio da aplicação da escala de Braden. A digitação dos dados dos questionários foi realizada em planilhas no programa Microsoft Excel e representados por meio de tabelas e gráficos.

A coleta de dados ocorreu nos meses de fevereiro a abril de 2013. As informações foram fornecidas pelos pacientes e pelos cuidadores. No momento da pesquisa a Unidade de Assistência Domiciliar constava com 189 pacientes ativos. Destes, um total de 124 foram categorizados, conforme anotações em prontuários, como pacientes totalmente dependentes para realização de AVD (Grau V na escala espanhola para avaliação de incapacidades funcionais). Destes, realizou-se a coleta com um total de 98 pacientes. Dos 26 pacientes restantes, 13 encontravam-se internados ou não estavam no domicílio quando da realização das visitas domiciliares, sete eram pacientes que realizavam algumas AVD, portanto, não faziam parte da população deste trabalho, quatro se recusaram a participar da pesquisa e dois pacientes eram crianças, desta forma, não foram incluídos neste trabalho.

A realização desta pesquisa foi precedida da aprovação pelo Comitê de Ética em Pesquisa da Universidade de Taubaté (SP) com o protocolo CEP/UNITAU no 390/12, de acordo com as diretrizes e normas regulamentadoras de pesquisa em seres humanos, Resolução CNS 196/96 ${ }^{8}$, assim como pela Supervisão de Saúde de Santo Amaro e Cidade Ademar, junto a Prefeitura do Município de São Paulo e pelo Comitê de Ética e Pesquisa da Secretaria Municipal de Saúde da Prefeitura Municipal de São Paulo. Os pacientes receberam o Termo de Consentimento Livre e Esclarecido, antes do início da coleta de dados.

\section{RESULTADOS}

O perfil epidemiológico é apresentado na Tabela 1.

Em relação a variável sexo, foram encontrados 57 pacientes do sexo feminino e 41 pacientes do sexo masculino. Quanto ao estado civil, a predominância ocorreu no atributo viúvo com 39 pacientes; outros 32 pacientes eram solteiros, 24 pacientes eram casados e três pacientes eram divorciados. Na apresentação da variável raça, um total de 67 pacientes se autodeclararam brancos, 15 pacientes se autodeclararam negros, 13 pacientes se autodeclararam pardos e três pacientes se autodeclararam amarelos.

As idades variaram conforme se segue: 18 pacientes possuíam entre 20 e 39 anos; 20 pacientes apresentavam entre 40 a 59 anos; 20 pacientes possuíam entre 60 a 79 anos e 40 pacientes apresentavam mais de 80 anos. A idade mínima 
foi de 20 anos e a máxima de 102. A média de idade foi de 66,3 anos e o Desvio Padrão foi de 23,67748.

A seguir, os dados sobre o tipo de cuidador, incontinência e presença de úlcera, estão apresentados na Tabela 2.

Do total de pacientes, 90 eram cuidados por familiares enquanto 8 eram cuidados por profissionais de saúde. Todos os pacientes apresentaram algum tipo de incontinência, havendo prevalência do quadro de incontinência anal e urinária associadas com um total de 85 pacientes; 13 apresentavam apenas incontinência urinária e nenhum paciente apresentou apenas incontinência fecal.

Houve prevalência das doenças do Sistema Nervoso com total de 42 ocorrências. $\mathrm{O}$ quadro mais frequente foi o de Paraplegia e Tetraplegia com 15 casos (34\%) seguido da doença de Alzheimer (DA) com 11 casos (25\%), resultados semelhantes ao encontrado por Chayamiti ${ }^{9}$ e Coelho et al..$^{10}$. Quanto às outras doenças, foram encontrados 35 casos de doenças do Sistema Circulatório. Todos os pacientes apresentaram Acidente Vásculo Encefálico (AVE), associado a Hipertensão Arterial Sistêmica (HAS). Também foi encontrado um total de sete

Tabela 1. Distribuição dos pacientes segundo Sexo, Estado Civil, Faixa Etária, Raça, São Paulo, SP, 2013.

\begin{tabular}{|c|c|c|}
\hline Variáveis & $\mathrm{n}$ & $\%$ \\
\hline \multicolumn{3}{|l|}{ Sexo } \\
\hline Masculino & 41 & 42 \\
\hline Feminino & 57 & 58 \\
\hline Total & 98 & 100 \\
\hline \multicolumn{3}{|l|}{ Estado Civil } \\
\hline Solteiro & 32 & 33 \\
\hline Casado & 24 & 24 \\
\hline Divorciado & 3 & 3 \\
\hline Viúvo & 39 & 40 \\
\hline Total & 98 & 100 \\
\hline \multicolumn{3}{|l|}{ Faixa Etária } \\
\hline $20-39$ anos & 18 & 19 \\
\hline 40-59 anos & 20 & 20 \\
\hline $60-79$ anos & 20 & 20 \\
\hline$>80$ anos & 40 & 41 \\
\hline Total & 98 & 100 \\
\hline \multicolumn{3}{|l|}{ Raça } \\
\hline Branca & 67 & 68 \\
\hline Negra & 15 & 16 \\
\hline Parda & 13 & 13 \\
\hline Amarela & 3 & 3 \\
\hline Outra & 0 & 0 \\
\hline Total & 98 & 100 \\
\hline
\end{tabular}

pacientes com quadro de Diabetes Mellitus (DM), sendo este um quadro secundário ao paciente estar acamado.

Quanto à pontuação da Escala de Braden e presença de UP, estes dados estão representados na Figura 1.

Dos pacientes em estudo, 82 pacientes apresentaram ausência de UP. Todavia, 16 pacientes encontravam em tratamento de UP. O valor mínimo da escala de Braden foi de 8 pontos e o valor máximo foi de 18 pontos, sendo a média de 13,04 pontos ( $\mathrm{DP}=2,363460539$ ).

Um total de sete pacientes apresentou pontuação equivalente ao Altíssimo Risco para o desenvolvimento de UP; destes, dois pacientes (29\%) apresentaram UP. Outros 36 pacientes apresentaram Alto Risco para o desenvolvimento de UP, sendo que três pacientes $(8,4 \%)$ apresentaram UP. Ao todo, 30 pacientes apresentaram Risco Moderado para o desenvolvimento de UP; destes, quatro pacientes (13\%) apresentaram UP. Um total de 25 pacientes apresentou Risco Baixo para o desenvolvimento de UP e sete pacientes (28\%) apresentaram UP. Nenhum paciente apresentou-se sem risco para o desenvolvimento de UP.

O Quadro 1 mostra os dados referentes a pontuação obtida com o uso da Escala de Braden nos pacientes. Este resultado pode ser melhor visualizado na Figura 2.

A subsescala percepção teve a média de $1,88(\mathrm{DP}=0,60)$ tendo como moda o valor 2. Observou-se predomínio da pontuação Muito Limitado ( $\mathrm{n}=61)$ seguido pela pontuação Totalmente Limitado $(n=24)$. A subescala umidade teve a média de 2,73 ( $\mathrm{DP}=0,68)$ tendo como moda o valor 3. Observou-se predomínio da pontuação

Tabela 2. Distribuição dos pacientes segundo Tipo de Cuidador Principal, Tipo de incontinência e Presença de UP, São Paulo, SP, 2013.

\begin{tabular}{lcc}
\hline Características & $\mathbf{n}$ & $\%$ \\
\hline Cuidador Principal & 90 & 92 \\
\hline Familiar & 8 & 8 \\
\hline Profissional de Saúde & 98 \\
\hline Total & 100 \\
\hline Tipo de incontinência & 13 & 13 \\
\hline Urinária & 0 & 0 \\
\hline Fecal & 85 & 87 \\
\hline Ambas & 98 & 100 \\
\hline Presençal & 16 & 16 \\
Sim & 82 & 84 \\
Não & 98 \\
\hline Total & 100 \\
\hline up: Úlcera por Pressão. &
\end{tabular}




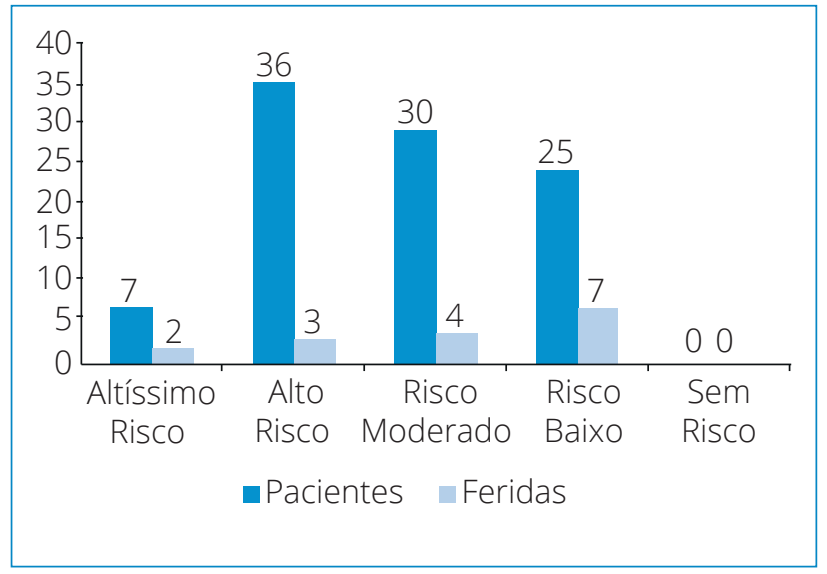

Figura 1. Pontuação da Escala de Braden e presença de Úlcera por Pressão. São Paulo, SP, 2013.
Ocasionalmente Molhada ( $\mathrm{n}=58$ ) seguido pela pontuação Muito Molhada ( $\mathrm{n}=27)$.

A subescala Atividade teve a média de 1,76 ( $\mathrm{DP}=0,70)$ tendo como moda o valor 2. Observou-se predomínio da pontuação Confinado a cadeira $(n=45)$ seguida pela pontuação Acamado ( $n=38)$. Essa pontuação está ligada diretamente as doenças de base na população estudada. A subescala Mobilidade teve a média de 1,80 (DP=0,63) tendo como moda o valor 2 . Observou-se um predomínio na pontuação Bastante Limitado $(\mathrm{n}=55)$ seguido pela pontuação Totalmente Imóvel $(\mathrm{n}=31)$.

A subescala Nutrição teve a média de 2,83 ( $\mathrm{DP}=0,62)$ tendo como moda o valor 3. Observou-se um predomínio da pontuação Adequado ( $n=64)$ seguido pela pontuação

Quadro 1. Subescalas da Escala de Braden e seus valores. São Paulo, SP, 2013.

\begin{tabular}{|lccccccc|}
\hline Categoria & Percepção & Umidade & Atividade & Mobilidade & Nutrição & Fricção & Total \\
\hline Média & 1,88 & 2,73 & 1,76 & 1,80 & 2,83 & 1,96 & 13,04 \\
\hline Mediana & 2 & 3 & 2 & 2 & 3 & 2 & 13 \\
\hline Moda & 2 & 3 & 2 & 2 & 3 & 2 & 12 \\
\hline Desvio Padrão & 0,6072 & 0,6818 & 0,7005 & 0,6366 & 0,6208 & 0,1731 & 2,3634 \\
\hline Mínimo & 1 & 1 & 1 & 1 & 1 & 1 & 8 \\
\hline Máximo & 3 & 4 & 3 & 3 & 4 & 2 & 18 \\
\hline
\end{tabular}

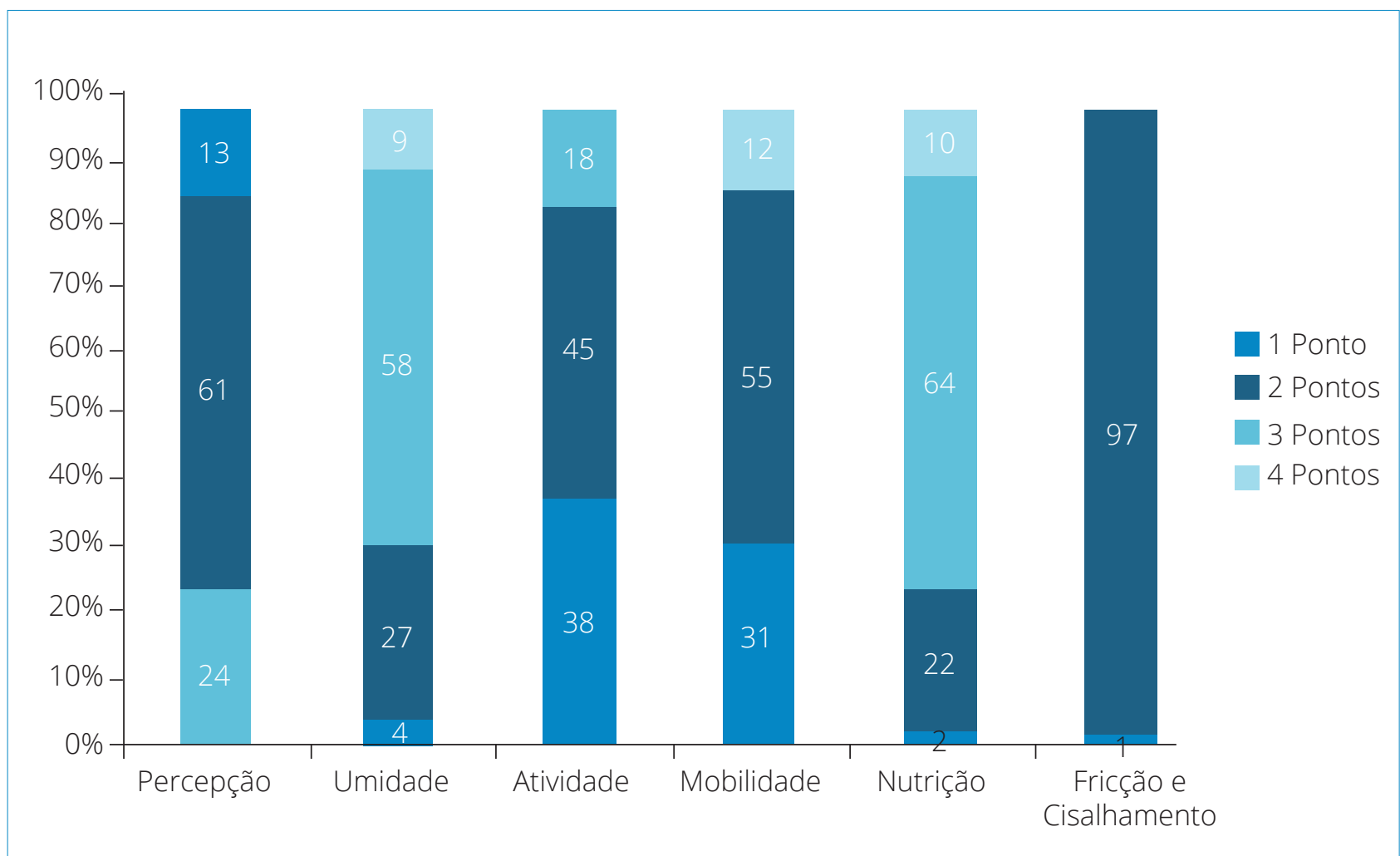

Figura 2. Pontuações das Subescalas da Escala de Braden. São Paulo, SP, 2013. 
Provavelmente Inadequado $(\mathrm{n}=22)$. A subescala Fricção e Cisalhamento teve a média de $1,96(\mathrm{DP}=0,17)$ tendo como moda o valor 2. Observou-se predomínio na pontuação Problema em Potencial ( $\mathrm{n}=97)$.

A seguir, a relação entre idade e escala de Braden, estão apresentados na Tabela 3.

Conforme a Tabela 3 observou-se que os menores resultados na Escala de Braden estão associados aos pacientes com idade superior a 60 anos, sugerindo que os idosos estão mais sujeitos ao surgimento das UP.Um total de 60 idosos apresentou risco para o desenvolvimento de UP, contra 38 pacientes com menos de 60 anos. Destes, sete idosos (43,75\%) apresentaram UP.

Dos 16 pacientes com feridas, um total de nove pacientes $(56 \%)$ possuía idade inferior a 60 anos; sete pacientes (44\%) apresentavam mais de 60 anos de idade.

\section{DISCUSSÃO}

Quanto ao sexo dos pacientes houve predomínio da população feminina, semelhante aos estudos de Chayamiti ${ }^{9}$, Coelho et al. ${ }^{10}$, Souza et al. ${ }^{11}$ e Menegon et al. ${ }^{12}$. A presença maior de mulheres quando comparado a de homens na maioria dos grupos etários, devido as maiores taxas de mortalidade experimentadas pelos homens, quanto pela maior longevidade feminina ${ }^{13}$.

Quanto ao estado civil, os viúvos foram maioria, seguidos dos solteiros. Este resultado difere do encontrado por Chayamiti', onde, em estudo semelhante, encontrou um total de $42,5 \%$ da população casada, $36,2 \%$ viúva, $14,9 \%$ solteira e 6,4\% como divorciado.

Em relação a distribuição das idades, houve predomínio dos pacientes idosos. Foram encontrados dados semelhantes em Chayamiti e Caliri ${ }^{14}$ sobre a média das idades dos pacientes. Evidenciou-se uma prevalência por parte dos

Tabela 3. Relação entre Pontuação da Escala de Braden e Idade. São Paulo, SP, 2013.

\begin{tabular}{|c|c|c|c|}
\hline \multirow{2}{*}{$\begin{array}{l}\text { Pontuação } \\
\text { Escala de Braden }\end{array}$} & \multicolumn{3}{|c|}{ Idade } \\
\hline & $<60$ anos & $>60$ anos & Total \\
\hline$<9$ & 2 & 5 & 7 \\
\hline 10 a 12 & 8 & 28 & 36 \\
\hline 13 a 14 & 14 & 16 & 32 \\
\hline 15 a 18 & 14 & 11 & 25 \\
\hline 19 a 23 & 0 & 0 & 0 \\
\hline Total & 38 & 60 & 98 \\
\hline
\end{tabular}

idosos (61\%) sendo que, destes, $67 \%$ apresentavam idade superior a 80 anos. Tais resultados são decorrentes da natureza da pesquisa assim como do público atendido pela Assistência Domiciliar, uma vez que as DCNT são mais frequentes com a população idosa ${ }^{15}$.

A maioria dos pacientes (68\%) se autodeclararam da raça branca. Chayamiti ${ }^{9} \mathrm{em}$ estudo semelhante, encontrou resultados análogos, com 85,1\% da população declarando-se branca.

A grande maioria dos pacientes tinha como cuidador um familiar. Resultados semelhantes foram obtidos por Chayamiti e Caliri ${ }^{14}$ em estudo sobre o tema. Foi encontrada uma grande quantidade de pacientes sob cuidados familiares como um fenômeno relacionado ao processo de transição demográfica da população ${ }^{2}$. Além disso, é sabido o baixo nível socioeconômico dos pacientes que não tem como despender gastos com a contratação de profissionais para a realização destes cuidados.

Todos os pacientes apresentaram quadro de incontinência, com predomínio do quadro associado de incontinência anal (IA) e incontinência urinária (IU). A IU é um sério problema de saúde pública sendo uma das mais recorrentes síndromes geriátricas e pode ser definida como a queixa de qualquer perda involuntária de urina ${ }^{16}$.

Houve predomínio das doenças do sistema nervoso, com destaque para os quadros de Paraplegia e Tetraplegia, assim como para a doença de Alzheimer. Foi identificada uma diferença dos resultados encontrados por Chayamiti e Caliri ${ }^{14}$ uma vez que seu estudo evidenciou um maior número de pacientes com doenças do sistema cardiovascular em relação às doenças do sistema nervoso. $\mathrm{O}$ mesmo acontece com o estudo de Rogenski e Kurcgant ${ }^{17}$, realizado em unidades de internação de um hospital onde houve predomínio das doenças cardiopulmonares em relação as doenças do sistema nervoso.

Houve uma prevalência de UP de 16\%, resultado semelhante à Chayamiti e Caliri ${ }^{14}$ onde foi encontrado um índice de prevalência de UP de 19,1\%, semelhante ao estudo em unidades de internação ${ }^{17}$, que encontraram uma prevalência de UP de 19,5\%. Contudo, em estudo com idosos sob assistência domiciliar ${ }^{10}$, encontrou-se uma incidência de UP de $31,4 \%$, quase o dobro do encontrado em nosso estudo. No mesmo estudo, os autores referem que apenas $10 \%$ dos pacientes apresentavam UP grau I, de modo que não houve equívocos de classificação de lesão por parte dos pesquisadores.

O valor mínimo da Escala de Braden foi de oito pontos e o máximo de 18 pontos. Estudo semelhante em ambiente hospitalar encontrou uma média de 13,86 para a Escala de 
Braden $(\mathrm{DP}=4,75)^{4}$. Outros estudos em serviços de assistência domiciliar encontraram médias de $13,32^{10}$ a $17,06^{9}$.

Estes resultados divergem daqueles encontrados por Chayamiti ${ }^{9}$ em relação ao risco dos pacientes para o desenvolvimento de UP, onde houve prevalência de pacientes com risco baixo $(48,9 \%)$, seguidos dos sem risco $(29,8 \%)$. Os pacientes com risco baixo foram apenas $4,2 \%$, evidenciando que estes se encontram em um risco menor de desenvolvimento de UP. Tais resultados vão ao encontro dos estudos de Coelho et al. ${ }^{10}$, Blanes et al. ${ }^{18}$ e França et al. ${ }^{19}$.

Apesar de todos os pacientes estarem em risco para desenvolvimento de UP, foi evidenciado que 84 pacientes não apresentavam lesões. Tal resultado pode ser interpretado como reflexo das atividades educativas, preventivas e de tratamento da equipe de assistência domiciliar, visando evitar o surgimento das lesões e complicações. Este resultado também pode ser atribuído a ação incansável dos cuidadores, em sua maioria absoluta familiares, frente aos cuidados destes pacientes e guiados pelas orientações da equipe de saúde.

A subescala Percepção teve predomínio de pacientes com sérias limitações para movimentação, com $87 \%$ dos pacientes. Tais resultados podem ser atribuídos devido a doença de base, no caso, predomínio das doenças nervosas e cardiovasculares que acarretaram sequelas motoras aos pacientes.

$\mathrm{Na}$ subescala Umidade houve predomínio das pontuações Ocasionalmente molhada e Muito Molhada. Estes resultados condizem com os valores encontrados para presença de IU (100\% dos pacientes). Foi identificado um maior valor para a pontuação Ocasionalmente Molhada devido a presença dos cuidadores e familiares, que, constantemente, estão prestando cuidados de modo a evitar as complicações.

A subescala Atividade teve predomínio das pontuações Confinado a Cadeira e Acamado, enquanto a subescala Mobilidade teve predomínio das pontuações Bastante Limitado e Totalmente Imóvel. Tais resultados podem ser associados à doença de base destes pacientes, assim como a presença de sequelas motoras nos mesmos.

Apesar disso, observou-se a adoção das medidas preventivas pelos cuidadores (utilização de superfície de suporte, uso de coxins, reposicionamento no leito a cada duas horas, hidratação corporal, troca de fraldas e medidas de higiene) além do que muitas lesões eram decorrentes da internação hospitalar. Nesse sentido, destaca-se a importância da educação continuada para a prevenção desse agravo, principalmente no contexto hospitalar.
$\mathrm{Na}$ subescala Nutrição o resultado Adequado predominou, fator este que auxiliaria na prevenção das UP. A má nutrição é considerada fator determinante na formação de UP, principalmente, para a diminuição da tolerância tissular à pressão. Estes resultados positivos nesta categoria se devem ao fato da grande maioria dos pacientes realizar alimentação via oral, além dos cuidadores e pacientes relatarem hábitos de alimentação e apetite preservados. Este resultado se contrapõe ao encontrado por Coelho et al. ${ }^{10}$, em estudo com idosos em atendimento domiciliar, onde foi evidenciado que $73,2 \%$ dos pacientes realizavam alimentação por via enteral. Todavia, um total de 22 pacientes apresentava a alimentação prejudicada, fato este que pode contribuir para o desenvolvimento de UP.

A subescala Fricção e Cisalhamento teve a maioria absoluta como Problema em Potencial. Menegon et al. ${ }^{12}$ encontraram um total de $52,9 \%$ de pacientes com a presença de Fricção e Cisalhamento, sugerindo que estes pacientes por serem dependentes para realização de AVD e estarem a maior parte do tempo confinados a cadeira ou leito, desta forma, expostos a estas forças que podem contribuir para o surgimento das UP.

Chayamiti ${ }^{9}$ encontrou um total de $22,2 \%$ de pacientes idosos com presença de UP, aproximadamente metade do encontrado neste estudo, conforme evidenciado na Tabela 3. Este resultado converge com o deste estudo e aponta para o problema da prevalência das UP na população idosa. A pessoa idosa apresenta maior incidência de UP devido às alterações na pele, tais como, diminuição da vascularização e de propriedades como percepção da dor e da resposta inflamatória, próprias da senescência. Também se verifica um aumento na probabilidade de doenças crônicas, as quais aumentam a susceptibilidade de desenvolvimento de úlceras por pressão.

\section{CONCLUSÃO}

Em relação ao perfil epidemiológico, houve predomínio da população feminina (58\%), viúvos (40\%), da raça branca (68\%) e $61 \%$ dos pacientes eram idosos e $40 \%$ eram octogenários.Os pacientes em sua maioria eram cuidados por familiares (88\%) e todos apresentaram algum tipo de incontinência, sendo mais frequente a associação de IA e IU (87\%). Houve predomínio das doenças do sistema nervoso (43\%), seguida pelas doenças do sistema circulatório (36\%). 
Em relação aos fatores que podem propiciar risco de desenvolvimento de UP todos os pacientes apresentaram risco para desenvolvimento de UP. Sete pacientes apresentaram Altíssimo Risco, 36 apresentaram Alto Risco, 32 apresentaram Risco Moderado e 25 apresentaram Baixo Risco. Nenhum paciente estava sem risco para o desenvolvimento de UP. Este cenário pode ser identificado devido ao quadro clínico dos pacientes, em situação de total dependência e a presença de IA e/ou IU nos pacientes estudados.

Quanto a análise das condições dos pacientes e sua classificação de risco para UP, veri ficou-se que 16 pacientes apresentaram UP. O escorre mínimo da escala de Braden foi de oito pontos e o máximo de 18 pontos, sendo que a média foi de 13,04 pontos ( $\mathrm{DP}=2,363460539)$. Os pacientes que mais apresentaram UP foram os classificados em Risco Muito elevado (29\%) e Risco leve (28\%). Sessenta pacientes com mais de 60 anos apresentavam risco para desenvolvimento de UP X 40 pacientes com menos de 60 anos. Destes, sete idosos (43,75\%) apresentaram UP.

Na subescala Percepção houve predomínio da pontuação Muito Limitado ( $\mathrm{n}=61$ ). Na subescala Umidade foi maior a pontuação Ocasionalmente Molhada $(\mathrm{n}=58)$. Na subescala Atividade predomíninou a pontuação Confinado à cadeira $(n=45)$. E na subescala Mobilidade a pontuação Bastante Limitado ( $\mathrm{n}=55)$ foi dominante. Já na subescala Nutrição a maior parte teve pontuação Adequado ( $\mathrm{n}=64)$, mas na subescala Fricção e Cisalhamento o predomínio foi na pontuação Problema em Potencial ( $\mathrm{n}=97)$.

\section{REFERÊNCIAS}

1. Organização Mundial de Saúde (OMS). Envelhecimento ativo: uma política de saúde. Brasília: Organização PanAmericana da Saúde; 2005.

2. Vieira CPB, Fialho AVM, Almeida PC, Moreira TMM. Idosos com acidente vascular encefálico isquêmico: caracterização sóciodemográfica e funcional. Rev Rene. 2012;13(3):522-30.

3. Thumé E, Facchini LA, Tomasi E, Vieira LAS. Assistência domiciliar a idosos: fatores associados, características do acesso e do cuidado. Rev Saúde Pública. 2010;44(6):1-10.

4. Gomes FSL, Bastos MAR, Matozinhos FP, Temponi HR, VelásquezMeléndez G. Avaliação de risco para úlcera por pressão em pacientes críticos. Rev Esc Enferm USP. 2011;45(2):313-8.

5. Serpa LF. Capacidade preditiva da subescala nutrição da escala de Braden para avaliar o risco de desenvolvimento de úlceras por pressão. [Tese]. São Paulo: Universidade de São Paulo, 2006.

6. Souza DMST, Santos VLCG. Fatores de risco para o desenvolvimento de úlceras por pressão em idosos institucionalizados. Rev Lat Am Enferm. 2007;15(5):958-64.

7. Pereira JMV, Cavalcanti ACD, Santana RF, Cassiano KM, Queluci GC, Guimarães TCF. Diagnósticos de enfermagem de pacientes hospitalizados com doenças cardiovasculares. Esc Anna Nery. 2011;15(4):737-45.

8. Brasil. Conselho Nacional de Saúde. Resolução n. 196 de 10 de Outubro de 1996. Diretrizes e normas regulamentadoras de pesquisa envolvendo seres humanos. Bioética. 1996;4(2):15-25.

9. Chayamiti EMPC. Prevalência de úlcera por pressão em assistência domiciliária em um distrito de saúde de Ribeirão Preto. [Dissertação de mestrado]. Ribeirão Preto: Universidade de São Paulo, 2008.

10. Coêlho ADA, Lopes MVO, Melo RP, Castro ME. O idoso e a úlcera por pressão em serviço de atendimento domiciliar. Rev Rene. 2012;13(3):639-49.
11. Souza JERB, Silva HF, Rabelo CBM, Bezerra SMG, Luz MHB, Rangel EML. Fatores de risco e ocorrência de úlcera por pressão em idosos institucionalizados. Rev Enferm UFPI. 2012;1(1):36-41.

12. Menegon DB, Bercini RR, Santos CT, Lucena AF, Pereira AGS, Scain SF. Análise das subescalas de Braden como indicativos de risco para úlcera por pressão. Texto Contexto Enferm. 2012;21(4):854-61

13. Lima e Silva EWN, Araújo RA, Oliveira EC, Falcão VTF. Aplicabilidade do protocolo de prevenção de úlcera por pressão em unidade de terapia intensiva. Rev Bras Ter Intensiva 2010;22(2):175-85.

14. Chayamiti EMPC, Caliri MHL. Ulcera por pressão em pacientes sob assistência domiciliária. Acta Paul Enferm. 2010;23(1):29-34

15. Silveira MM, Pasqualotti A, Colussi EL. Prevalência de doenças crônicas e prática de atividade física em adultos e idosos. Rev Bras Promoç Saúde. 2012;25(2):209-214.

16. Caldas CP, Conceição IRS, José Rita MC, Silva BMC. Terapia comportamental para incontinência urinária da mulher idosa: uma ação do enfermeiro. Texto Contexto Enferm. 2010;19(4):783-8

17. Rogenski NMB, Kurcgant P. Avaliação da concordância na aplicação da Escala de Braden interobservadores. Acta Paul Enferm. 2012;25(1):24-8.

18. Blanes L, Duarte IS, Calil JA, Ferrreira LM. Avaliação clínica e epidemiológica das úlceras por pressão em pacientes internados no hospital São Paulo. Rev Assoc Med Bras. 2004;50(2):182-7.

19. França SPS, Melo JS, Araújo LS. Risco de desenvolvimento de úlcera por pressão em idosos. Rev enferm UFPE. 2013;7(1):755-62. 